\title{
Reviews
}

\section{Regional anaesthesia for outpatient surgery}

\author{
S K Pandit, U A Pandit
}

University of Michigan School of Medicine, Ann Arbor, Michigan, USA

\begin{abstract}
Regional anaesthesia is an ideal anaesthetic technique for many outpatient surgery procedures. With proper preparation, selection and planning, regional anaesthesia will provide excellent operating conditions for the surgeon and a very satisfactory anaesthetic state for the patient in a cost-effective way. Its minimum effect on major organ functions, intense sensory blockade, low potential for postoperative side effects, and its ability to provide postoperative pain control, offers an excellent set up for these patients who will go home soon after the surgery.
\end{abstract}

Key words: Anaesthesia, outpatient, ambulatory, regional; surgery, outpatient

The most common form of anaesthesia used for outpatient surgery is general anaesthesia. It is preferred over regional anaesthesia by both surgeons and patients in this setting. Surgeons prefer general anaesthesia because they consider its initiation faster, which means less 'waste of time' before starting the operation. Patients often prefer general anaesthesia as a result of anxiety about the surgery and a feeling that going to sleep for the procedure will 'solve the problem'. In addition, many patients have concerns that are based on misinformation about the complications of regional anaesthesia. The majority of practising anaesthesiologists prefer general anaesthesia for outpatient surgery because they believe that general anaesthesia provides better comfort and safety for the patient and better surgical conditions for the surgeon. Some anaesthesiologists dislike regional anaesthesia because they lack confidence in their own ability to perform a successful block in an expeditious way. Others are overly concerned about the potential side effects of regional anaesthesia (e.g. postdural puncture headache and backache, prolonged motor blockade, and delay in discharge due to inability to void after spinal or epidural anaesthesia).

\section{Possible advantages of regional anaesthesia for outpatient surgery}

The primary goal of outpatient anaesthesia is to provide an anaesthetic that is safe and effective, can be adminis-

\footnotetext{
Accepted: 15 June 1994

Correspondence and reprint requests to: SK Pandit, Department of Anesthesiology, Box \#0048, University of Michigan Medical Center,
} Ann Arbor, MI 48109-0048, USA

C 1994 Butterworth-Heinemann Ltd 0966-6532/94/030325-11
Table 1. Possible advantages of regional anaesthesia over general anaesthesia

Less physiological trespass, less depressant effects on major organ functions (e.g. cardiovascular, hepatic, renal, respiratory, metabolic)

Use of less pharmacological agents

Quicker recovery of physiological and psychomotor functions and shorter discharge time

Patient participation in the evaluation of intraoperative surgical pathology and therapeutic options (via video)

Quicker diagnosis of certain intraoperative complications (e.g. pneumothorax or pneumo-mediastinum during laparoscopic surgery)

Less postoperative side effects (e.g. nausea and vomiting, sore throat, muscle pain, dizziness)

Better control of immediate postoperative pain

More cost-effective

tered expeditiously, provides an appropriate surgical condition with minimum effects on organ functions and minimum side effects, and ensures a quick return of normal physiological and psychomotor functions soon after the operation.

With the proper selection of patients, surgical procedures, anaesthetic techniques, local anaesthetic agents and adjuvants, regional anaesthesia can be a safe and effective alternative to general anaesthesia and very satisfying for the anaesthesiologist and the patient. Table 1 lists the possible advantages of regional anaesthesia over general anaesthesia.

Regional anaesthesia may indeed be the ideal anaesthetic for outpatient operations in many situations. It anaesthetizes only a part of the body, with limited depression of major organ function and there may also be some advantages in having the patient awake during certain types of surgery. For example, with endoscopic 
surgical techniques and instant video screening of surgical pathology, surgeons can involve an awake patient under regional anaesthesia in decision-making about surgical options.

\section{Are the objections against regional anaesthesia for outpatient surgery valid?}

Many of the objections against regional anaesthesia for outpatient surgery are not valid. The issues involved in these objections can be addressed by considering the following questions:

1. Does the use of regional anaesthesia hamper the efficiency of the outpatient surgery facility?

2. Is regional anaesthesia necessarily more anxiety-provoking for the patient?

3. Does regional anaesthesia provide good surgical conditions and patient comfort?

4. Should occasional failure of regional anaesthesia preclude its use for outpatient surgery?

5. Are there more intraoperative and postoperative side effects after regional anaesthesia compared to general anaesthesia?

6. Is regional anaesthesia cost effective?

Does the use of regional anaesthesia hamper the efficiency of the outpatient surgery facility?

Operating room turn-over time

In today's outpatient surgery units the top administrative priority seems to be rapid and efficient turn-over of cases. With a quick turn-over of cases, administrators, surgeons, nurses, and anaesthesiologists all benefit. The opponents of regional anaesthesia argue that the initiation of regional anaesthesia not only takes a longer time than general anaesthesia, but there is also a chance of failure with the regional technique. These problems can hamper a quick turn-over of cases.

Not all regional anaesthetic techniques take a longer time to initiate. In experienced hands, for example, a subarachnoid block should take no longer to initiate than general anaesthesia. For an anaesthesiologist who is trying to do all the cases in one operating room singlehandedly, however, initiation of certain slow-onset types of regional anaesthesia, such as brachial plexus block or epidural anaesthesia, may indeed take longer than general anaesthesia. So, whenever possible these blocks should be initiated ahead of time in a different room (anaesthesia induction room or holding room) by a second anaesthesiologist. Once regional anaesthesia is established and tested ahead of time, the operation can be started in less time than is required to initiate general anaesthesia. It also takes less time to get the operating room ready for the next case when regional anaesthesia is used. This is due to fewer disposable items needing to be replaced between cases. Furthermore, patients receiving regional anaesthesia are ready to be transferred as soon as the operation is completed whereas a patient under general anaesthesia must be awakened, pharmacologi- cally reversed, extubated, and the airway safety ensured before the patient can be transferred to the postanaesthesia care unit (PACU). This makes the turn-over time even shorter for regional anaesthesia patients. Thus, when properly planned and selected, the operating room turn-over time should not be any longer for regional anaesthesia as compared to general anaesthesia and it can be shorter.

\section{PACU turn-over time}

Do patients who receive regional anaesthesia stay in the recovery room longer than those who receive general anaesthesia for the same operation? This is likely only if the patient has had a prolonged sympathetic, motor and sacral parasympathetic block and is unable to walk or void soon after the operation. Prolonged motor block and sacral parasympathetic block can be avoided by carefully selecting the type and dose of local anaesthetic and additives used. Avoidance of inappropriate local anaesthetic (e.g. tetracaine, etidocaine, bupivacaine) and inappropriate additives (e.g. epinephrine), will reduce the PACU stay after regional anaesthesia. Also, since regional anaesthesia causes less nausea and vomiting and less uncontrolled postoperative pain compared to general anaesthesia and the patient needs less medication to control these side effects, discharge criteria are met earlier and the patient can be discharged sooner. Several studies have shown that recovery time is actually shorter after regional anaesthesia compared to general anaesthe$\operatorname{sia}^{1-4}$.

\section{Is regional anaesthesia necessarily more anxiety- provoking for the patient?}

It is to be expected that every patient will be anxious before an operation to a greater or lesser extent. It is the duty of the healthcare professionals, especially the anaesthesiologist, to prepare the patient appropriately both psychologically and pharmacologically before the operation to reduce anxiety. Patients who are anxious about regional anaesthesia believe they cannot handle the additional strain of the 'needlesticks' for regional anaesthesia and of staying awake during the operation. Inappropriate anxiety, however, can be relieved with proper education, explanation and reassurance and by the appropriate use of anxiolytics, sedatives, analgesics and amnesics.

Some patients are unduly fearful about the complications of regional, especially spinal anaesthesia. These concerns include: fear of getting permanently paralyzed, prolonged and severe headache, backache and the necessity of lying flat in bed for a prolonged time. Sometimes these concerns are fixed in patients' minds and cannot be easily changed. In others, concerns can be dispelled by education about the recent advances in technology, about new spinal needles, about newer and shorter-acting medications and by citing the results of many large studies showing the safety of regional anaesthesia ${ }^{5-9}$. Educating patients about causes, prevention and treat- 
ment of postdural puncture headache, and providing reassurances that they do not have to stay flat in bed after the operation is also very important.

It is a common observation that patients usually reject regional anaesthesia when approached by an inexperienced anaesthesiologist and often accept it readily when approached by an experienced and confident anaesthesiologist. A Preanaesthesia Clinic visit or prior visit to the anaesthesiologist may serve to reduce patient anxiety and dispel misunderstandings about the complications of regional anaesthesia. In most situations, after a short supportive conversation, the patient feels comfortable about regional anaesthesia. Many patients are simply unaware of the many advantages of regional anaesthesia (i.e. less nausea and vomiting, better pain control, less physiological trespass) and of the fact that they do not necessarily have to be totally awake during the procedure.

On the other hand, it is unwise to force regional anaesthesia upon unwilling patients and upon those who are severely anxious about needles and unduly concerned about the side effects of regional anaesthesia. In addition to the psychological and pharmacological preparation of the patient, judicious use of an intravenous anxiolytic agent, such as midazolam $1-2 \mathrm{mg}$ iv, often works wonders in transforming an overly anxious patient into a cooperative and smiling subject during the placement and conduct of a regional block. There are some indications that pretreatment with a benzodiazepine may increase the threshold for local anaesthetic-induced systemic toxicity ${ }^{10}$. In young children regional anaesthesia should be initiated after the child is asleep.

\section{Does regional anaesthesia provide good surgical conditions and patient comfort?}

Some surgeons dislike regional anaesthesia, believing that it may not provide good surgical relaxation. Since very few outpatient operations require intense muscle relaxation, this is not a valid objection against regional anaesthesia. Inadequate analgesia or so-called 'patchy block' however, may make the patient complain of pain and discomfort and prevent the surgeon from getting a quiet field of operation. Preoperative preparation, explanation of what to expect, and judicious use of pharmacological agents including analgesics and sedatives and even light general anaesthesia should obviate most of these problems.

To reap the benefits of regional anaesthesia, both the anaesthesiologist and the surgeon must be willing to modify their practice pattern to suit the conditions offered by regional anaesthesia. Indeed, the surgeon's cooperation and support is essential if regional anaesthesia is to be successful at a given outpatient surgery centre. The anaesthesiologist must therefore take the lead in educating the surgeon and nurses with data about the situations where regional anaesthesia will benefit the patient.
Should occasional failure of regional anaesthesia preclude its use for outpatient surgery?

A major reason many anaesthesiologists are reluctant to use regional anaesthesia is that, unlike general anaesthesia which 'always works', there is a recognizable failure rate for regional anaesthesia. The failure rate depends to a large extent on the experience of the anaesthesiologist and the choice of the appropriate regional block for the patient and for the surgical procedure. It is to be expected that it will sometimes be impossible to perform, and at other times regional anaesthesia alone will not be enough for the surgery, but an experienced anaesthesiologist is usually able to reduce the failure rate to a minimum. Planning ahead is one key to success; plan to place the block ahead of time rather than be rushed into it at the last moment. A so-called 'failed' regional block can be converted into a 'successful anaesthetic' by supplementation with sedatives (e.g. midazolam, propofol), systemic analgesics (e.g. fentanyl, alfentanil or remifentanil), additional nerve blocks (e.g. ulnar and median nerve blocks after axillary block), or light general anaesthesia (e.g. propofol, inhaled anaesthetics). Small analgesic doses of ketamine $\left(1.0 \mathrm{mg} \mathrm{kg}^{-1}\right.$ or less, iv) often work well to make inadequate block successful ${ }^{1}$.

\section{Are there more intraoperative and postoperative side effects after regional anaesthesia compared to general anaesthesia?}

\section{Intraoperative side effects}

Although regional anaesthesia usually poses fewer intraoperative problems than a general anaesthetic technique, some specific side effects should always be kept in mind. The side effects of regional anaesthesia include arterial hypotension and bradycardia following central neuronal block, pneumothorax after certain types of blocks, systemic toxicity and allergic reactions to local anaesthetics.

\section{Hypotension and bradycardia}

Since the mechanism of hypotension and bradycardia following high spinal or epidural anaesthesia is well known, its prevention and management is fairly simple. Judicious and prompt administration of intravenous fluids and an appropriate vasopressor therapy should remedy the situation. When doing either spinal or epidural anaesthesia in young healthy patients, one should always be ready to treat the rare case of severe sudden bradycardia, or even cardiac arrest with aggressive resuscitative measures, including early use of epinephrine ${ }^{12}$. Fortunately, the majority of outpatient operations can be performed with either a low spinal or low epidural block, which does not involve profound sympathetic denervation and rarely poses serious problems.

\section{Pneumothorax}

The regional blocks that have higher risks of pneumothorax are supraclavicular and interscalene brachial 
plexus blocks and intercostal blocks. These are usually avoided for outpatient surgery unless their potential benefits outweigh the risks.

\section{Systemic toxicity and allergic reactions}

Systemic toxicity is always a possibility during regional anaesthesia. This occurs when either an intravascular (venous or arterial) injection is made, or a gross overdose of the local anaesthetic is given. Prevention of intravascular injection by careful aspiration for blood before each incremental dose is injected and careful avoidance of an overdose are the best methods to prevent this complication. One should always be on the lookout for symptoms and signs of systemic toxicity (i.e. change in mental status, twitching and convulsions, hypotension, arrhythmias) and be ready to treat and resuscitate with oxygen, hyperventilation, benzodiazepine, succinylcholine and physiological support. The central nervous system toxicity is a self-limiting process, the plasma level of the local anaesthetic drops quickly as it gets cleared by the liver, so the main emphasis is to keep the patient well oxygenated and ventilated for the short time it requires for the symptoms to stop. Cardiotoxicity, which may occur after bupivacaine, needs to be aggressively treated with epinephrine and antiarrhythmic agents. Allergic reactions are unusual, but can occur after the ester type of local anaesthetics (procaine, chloroprocaine) and are very rare after the amide types (lidocaine, prilocaine, bupivacaine).

Standards for intraoperative patient monitoring and preparation during regional anaesthesia should be the same as for general anaesthesia, as recommended by the American Society of Anesthesiologists Standards for Basic Anesthetic Monitoring ${ }^{13}$.

\section{Postoperative side effects}

Outpatient surgery patients differ from inpatients because they must be ready to go home soon after the operation, and they do not have a trained healthcare professional at home to detect and take care of any complications that may arise. So, it is imperative that outpatients be educated before surgery about possible postoperative side effects and complications, and the attendant has a clear understanding of what to do should they occur.

\section{Anaesthesia-related side effects in Post Anaesthesia Care Unit ( $P A C U$ )}

Certain anaesthesia-related side effects are more common after general anaesthesia than regional anaesthesia. They are: nausea and vomiting, unrelieved postoperative pain, drowsiness, dizziness, malaise ${ }^{2,4,14}$. Postoperative pain, especially immediate pain, can be controlled more easily if the patient has received regional anaesthesia during the operation. More drowsiness is to be expected after general anaesthesia than after regional anaesthesia, unless there has been excessive use of intraoperative and postoperative sedatives and other central nervous system (CNS) depressants during regional anaesthesia.

Inability to ambulate, inability to void

Other immediate postoperative side effects that may be more common after central neuroaxial blocks, like spinal and epidural anaesthesia, than after general anaesthesia are: possible delay in the ability to ambulate, delay in the ability to void, and backache. The inability to ambulate is related to the motor weakness and possibly the orthostatic hypotension due to sympathetic block that occurs with the central neuronal blocks, and the amount of CNS depressants the patient has received. Inability to void is usually related to the prolonged sacral parasympathetic block and possible atony of the bladder following prolonged distention of the bladder after autonomic block.

The incidence of these side effects can be greatly minimized by the proper choice a short-acting local anaesthetic agent and additives. Randel and colleagues ${ }^{4}$ found discharge time after knee arthroscopy was shorter after epidural anaesthesia compared to general anaesthesia. Epinephrine as an additive should not be used without consideration of its ability to prolong the block and possible consequence (e.g. delayed discharge). The use of epinephrine with local anaesthetic for spinal anaesthesia and excessive intravenous hydration should be avoided for elderly males as they are often associated with a delay in voiding in these patients.

\section{Postoperative side effects after discharge}

Common anaesthesia-related side effects that occur after discharge and at home are nausea and vomiting, unrelieved pain, malaise, drowsiness, dizziness, muscle pain, backache and headache. Among these, only headache and backache are more common after central regional blocks than after general anaesthesia.

\section{Postdural puncture headache (PDPH)}

Postdural puncture headache is the most important concern following spinal anaesthesia and to some extent after epidural anaesthesia. PDPH is a unique and characteristic type of headache. It usually appears $24-72 \mathrm{~h}$ after the dural puncture; the headache may involve the occiput, frontal region and the neck, and it is postural in nature, i.e. it is made worse during sitting or in an erect position and is relieved on reclining and by abdominal pressure. It is often associated with other symptoms like nausea and diplopia. The headache occurs due to the leakage of cerebrospinal fluid (CSF) through the hole in the dura created by the spinal or epidural needle. The severity and the duration of the symptoms depend on the size of the hole/s and the existing CSF pressure in the lumbar region. The headache is more common in young individuals, especially females.

Contrary to the commonly held belief ${ }^{15-17}$ that early postoperative ambulation after spinal anaesthesia has no effect on the incidence of PDPH, it has recently become 
obvious that the incidence of PDPH is higher after outpatient than inpatient surgery, especially in young patients. A few papers from Europe $\mathrm{e}^{18-20}$ claim that the incidence of PDPH following spinal anaesthesia in young patients undergoing outpatient surgery is unacceptably high and may be as high as $37 \%$. Reports from the United States ${ }^{21-23}$, including studies by Perz, Mulroy, and Neal, supported the conclusion that the incidence of PDPH after surgery in young outpatients is indeed higher than in inpatients, but in the order of $5-10 \%$.

Following the publication of these reports, most anaesthesiologists restricted their use of spinal anaesthesia only to older patients ( $>50 \mathrm{yr}$ ) scheduled for outpatient surgery.

The use of finer needles ${ }^{24,25}$, directing the bevel of the needle longitudinally to the fibres of the dura rather than transversely ${ }^{26}$, and the use of the lateral rather than the midline approach ${ }^{27}$, are some of the measures advocated to reduce the incidence of PDPH. Until the introduction ${ }^{28.29}$ and recent widespread adoption of the non-cutting pencil-point spinal needles (i.e. Whitacre and Sprotte needles), no significant difference in practice habits were noticed. With the increasing use of these needles, especially 25 or 27 gauge Whitacre needles, the incidence of PDPH has dropped sharply and spinal anaesthesia for outpatient surgery has become acceptable for all age groups ${ }^{30-33}$. At present, the overall incidence of PDPH following spinal anaesthesia in young patients is less than $2 \%$ or about the same as after epidural anaesthesia. Besides this, the severity of headache after spinal anaesthesia (due to smaller and less traumatic dural puncture) is likely to be less than after epidural anaesthesia. As a result, the fear of PDPH following spinal anaesthesia is no longer a relevant reason to avoid this useful technique for outpatient surgery.

\section{Backache}

Brown reported that backache occurs after spinal, epidural and even after general anaesthesia ${ }^{34}$. Backache is usually related to the prolonged recumbency during and after an extensive surgery, but is certainly made worse by the needlesticks in the back (sometimes multiple) during the performance of the regional block. Trauma to the ligaments, periosteum and bone during placement of spinal and epidural anaesthesia are the likely causes. Since thicker needles are used for epidural compared to spinal anaesthesia, epidural anaesthesia is associated with more backache ${ }^{4}$. Backache, when it occurs, is self-limiting and usually lasts for a few days. Simple analgesics and bed rest are recommended when needed.

A different kind, or more severe, backache after epidural anaesthesia that has been associated with the use of chloroprocaine has been reported ${ }^{35,36}$. This backache may be quite incapacitating requiring hospitalization and treatment with narcotics. Studies have shown that the incidence and severity of backache is related to the volume ( $>25 \mathrm{ml})$ and repeated injections of chloroprocaine (Nesacaine MPF) ${ }^{37,38}$. The possible mechanisms of this localized severe muscle pain include direct muscle irritation by the low $\mathrm{pH}$ of chloroprocaine and the calcium chelating effect of the preservative disodium ethylene diamine tetra-acetic acid (EDTA), an antioxidant ${ }^{39}$.

\section{Permanent neurological complications}

The concerns about permanent neurological complications following spinal anaesthesia are widespread among patients. Direct trauma to neural tissues, ischaemic or chemical injury to the spinal cord, compression of the spinal cord following epidural haematoma or abscess and infection are some of the dreaded possible complications of regional anaesthesia. These complications must be kept in mind and all possible preventative steps taken. Unintentional injection of chloroprocaine into the subarachnoid space has been reported to cause permanent neurological deficits (cauda-equina syndrome). Low $\mathrm{pH}$ and the preservative, sodium bisulfite, in the formal preparation of commercially available chloroprocaine were thought to be the cause of this complication ${ }^{40,41}$. Fortunately several large studies ${ }^{5-9}$ have shown that the incidence of permanent neurological complications following spinal and epidural anaesthesia are extremely rare.

\section{Is regional anaesthesia cost-effective?}

There is little disagreement that regional anaesthesia is a cost-effective form of anaesthesia care for outpatient surgery. A successful regional block, which can be accomplished with a single agent provides most of the components of a balanced anaesthetic regimen: intraoperative analgesia (sensory block); muscle relaxation (abolition of muscle tone, and occasionally motor blockade); control of sympathetic overactivity (sympathetic block); and postoperative pain control. Hypnosis or anxiolysis, when needed, can be provided by systemic agents. The cost of the local anaesthetic to produce the anaesthetic state by regional anaesthesia is much less than that of general anaesthesia. The drugs needed for general anaesthesia include intravenous agents, volatile and gaseous agents, narcotic analgesics, muscle relaxants, reversal agents and many other adjuvants. All these are expensive. There are fewer indirect costs with regional anaesthesia when all the disposable anaesthesia items are needed for general anaesthesia are considered. Furthermore, in the postoperative period there is less need for analgesics and antiemetics after regional anaesthesia. Among other indirect costs, there is a saving in operating room (OR) time and PACU time because of the quicker $\mathrm{OR}$ and PACU turn-over with regional anaesthesia.

\section{Indications of regional anaesthesia for outpatient surgery}

Regional anaesthesia is most appropriate for operations on the extremities, surface operations on face, trunk, perineum, and some limited pelvic and abdominal operations. Common outpatient operations performed under regional anaesthesia are: orthopaedic operations 
of upper and lower extremities (e.g. knee arthroscopic surgery, hand surgery); gynaecological surgery of the perineum (e.g. cone biopsy, D\&C); general surgical procedures on body surface (e.g. hernia repair, breast biopsy, excision of lumps and bumps); a variety of plastic surgery procedures; most ophthalmic surgery procedures; and some otolaryngeal procedures.

\section{Regional anaesthesia for laparoscopic surgery}

Laparoscopic surgery, one of the commonest outpatient procedures, is not usually performed under regional anaesthesia, because significant shoulder discomfort may be present when the abdomen is overdistended with insufflating gas and the patient is placed in a steep Trendelenburg position. In this position, unless ventilation is controlled, respiratory functions may be compromised, especially in obese patients. Increased intraabdominal pressure may encourage gastric regurgitation and pulmonary aspiration.

Nevertheless, in some centres in the United States, regional anaesthesia is routinely used for these procedures $^{2,3}$ with great success. If the patient is motivated and the surgeon is gentle, skilful, and can do the surgery with minimal distention of the abdomen with only a minimum Trendelenburg position in a relatively short time ( $30 \mathrm{~min}$ or so), spinal or epidural anaesthesia can be successfully and advantageously used for pelvic laparoscopic procedures. Use of nitrous oxide in place of carbon dioxide as the insufflating gas is better tolerated by the awake patient. A high sensory block, up to a level of $\mathrm{T}_{2-3}$ thoracic segment, is necessary for the comfort of the patient. Spinal anaesthesia with lidocaine and fentanyl provides more intense analgesia and better muscle relaxation than epidural anaesthesia and may be the preferred regional block for pelvic laparoscopic surgery.

\section{Characteristics of local anaesthetic agents and adjuvants suitable for outpatient surgery}

Selection of appropriate local anaesthetic agents and adjuvants for regional anaesthesia for outpatient surgery will make all the difference between failure or success. Most of the common problems that arise are due to the wrong choice of local anaesthetic agent, especially for spinal or epidural anaesthesia, and to some extent choice of the wrong regional anaesthetic technique. For example, a prolonged stay in the PACU and prolonged discharge time, delayed return of motor function after the operation, delayed voiding after the operation, and to some extent backache after epidural anaesthesia, can in some cases be ascribed to the wrong choice of local anaesthetic agent or the adjuvant used and possibly to the use of the wrong dosage.

\section{Local anaesthetic agents}

Traditionally, for inpatient operations, it is better that the regional block lasts half an hour longer than the expected duration of the operation rather than a few minutes too short. So the traditional recommendations have always been to choose a longer-acting agent 'just in case' the operation lasts longer than expected.

For outpatient surgery, however, a longer-acting local anaesthetic agent for spinal and epidural anaesthesia should not automatically be selected (to cover an unexpectedly longer duration of surgery). Instead, one should select an agent to fit the expected duration of the operation and remain prepared to supplement with systemic analgesics or with light general anaesthesia, if the operation lasts longer. With the advent of propofol, a quickacting intravenous agent and the quick-acting synthetic narcotics, fentanyl, alfentanil, remifentanil, with a short duration of action, has made these decisions easier. If this plan is not feasible, then the use of a continuous epidural technique should be considered, so that a shortacting agent may be used by intermittent injections or by a continuous infusion via a syringe pump.

The local anaesthetic agents that are suitable for outpatient surgery are either short-acting agents (e.g. procaine, chloroprocaine) or intermediate-acting agents (e.g. lidocaine, prilocaine and mepivacaine). Although long acting, bupivacaine and ropivacaine should be used very rarely; they may be used for peripheral or caudal blocks in children. Extra-long-acting drugs like tetracaine and etidocaine are contraindicated for outpatient surgery.

\section{Dextrose}

Dextrose (glucose) is added to the local anaesthetic solution for spinal anaesthesia to make the solution hyperbaric in relation to CSF. Hyperbaric solution allows the anaesthesiologist to direct the local anaesthetic to the desired spinal segments, either to restrict the extent of the block (e.g. 'saddle block') or to increase the extent of the block to the thoracic segments, without greatly reducing or increasing the amount of drug or the volume used. The age-old practice of adding glucose to the local anaesthetic, especially to lidocaine, has recently been questioned ${ }^{42,43}$. A high concentration of glucose $(10 \%)$, because of its hyperosmolarity can be neurotoxic. A few case reports of neurotoxicity (cauda-equina syndrome) following the intermittent injection technique via a spinal catheter has been implicated to glucose-containing lidocaine $^{44-46}$. A study by Ross suggests that a large local accumulation of the repeatedly injected lidocaine with dextrose combined with a minimum spread to the other segments probably caused the neurotoxicity ${ }^{47}$. The controversy about dextrose-containing lidocaine for single shot spinal anaesthesia is new and is not likely to be resolved soon. Several authors are re-exploring the possibility of using plain lidocaine $2 \%$, bupivacaine $0.5 \%$ or procaine $2.5 \%$, without dextrose for spinal anaesthesia in isobaric conditions with good results ${ }^{48}$. Alternatively, a lower concentration of dextrose (less than $7.5 \%$ ) may be tried. 


\section{Epinephrine}

There are several reasons why epinephrine is added to the local anaesthetic agent. It is used primarily to increase the duration of the block, but it is also used to reduce the peak plasma level of the local anaesthetics (reduced chance of systemic toxicity) and to improve the quality of the block. While the addition of epinephrine (1:200000-1:400000 concentration) to the local anaesthetic is quite appropriate for outpatient surgery on some occasions, one must be very careful about its use in the elderly, diabetics and in patients with arteriosclerosis. Inappropriate use of epinephrine may give rise to prolonged motor, sensory and autonomic block and a delay in discharge time.

\section{Bicarbonate}

Bicarbonate is often added to the local anaesthetic agent to hasten its onset of action ${ }^{49}$. Addition of bicarbonate increases the $\mathrm{pH}$ of the local anaesthetic agent and thus increases the non-ionized portion of the local anaesthetics in the mixture which can penetrate the cell membrane faster. The addition of bicarbonate $\left(1 \mathrm{mEq} 10^{-1} \mathrm{ml}\right)$ makes the most difference in local anaesthetic agents which have a lower $\mathrm{pH}$, (e.g. chloroprocaine, lidocaine and mepivacaine). However, the reduction of the onset time is not of much clinical significance, especially if the regional block is planned and performed ahead of time. The $\mathrm{pH}$ adjustment of bupivacaine and etidocaine is not supported in studies by DiFazio ${ }^{50}$ and because of the limited base solubility (precipitation) this practice may be dangerous. Therefore addition of bicarbonate, as an additive to the local anaesthetic, has limited clinical value in outpatient anaesthesia practice.

\section{Narcotics}

Narcotics are often added to the local anaesthetic mixture to augment the sensory blockade of spinal and epidural anaesthesia without increasing the degree of the autonomic blockade. Fentanyl (a $\mu$ agonist) is the most common narcotic used for this purpose. Sufentanil may also be used. Addition of fentanyl to the local anaesthetic agent seems to provide a more profound analgesia for the intraoperative period and more prolonged analgesia for the immediate postoperative period.

When used in moderate doses, epidural or subarachnoid fentanyl is safe for outpatient use. The dose of fentanyl as adjuvant to spinal anaesthetic is: $10-25 \mu \mathrm{g}$, and for epidural anaesthesia 50-100 $\mu \mathrm{g}$. With these doses, the minor side effects of intraspinal narcotics, such as pruritus, and nausea and vomiting, are rarely seen; and delayed respiratory depression, the major side effect, is unknown. The longer-acting narcotics like morphine and meperidine, however, should not be used for spinal or epidural injection during outpatient surgery. Whether intra-articular injection of morphine with bupivacaine provide additional postoperative analgesia following knee arthroscopy is still controversial ${ }^{51.52}$.

\section{Appropriate regional anaesthetic blocks for outpatient surgery}

Apart from the various forms of infiltration blocks, the common regional anaesthetic techniques used for outpatient surgery are intravenous regional anaesthesia (IVRA), axillary brachial plexus block and spinal, epidural and ankle blocks. Less commonly-used regional anaesthetic techniques for outpatient surgery are wrist, elbow, knee, popliteal and femoral-sciatic block. Other common and valuable blocks, especially for children, are: ilio-inguinal and ilio-hypogastric, penile and caudal blocks. For ophthalmic operations, retrobulbar and peribulbar blocks are universal.

Technical details of how to perform these blocks and doses of the appropriate local anaesthetic agents have been described in many text books $s^{53.54}$. A brief description of a few common blocks for outpatient surgery follows.

\section{Intravenous regional anaesthesia (IVRA)}

Intravenous regional anaesthesia is one of the most common regional anaesthetic blocks performed for outpatient surgery. This block is most suitable for operations on the upper extremities, especially hand surgery, which lasts for less than 90-120 min. It can also be used for operations on the lower extremities. The advantages of IVRA are that it is simple to perform, has a quick onset and offset of action and usually provides good surgical conditions. Additionally, when used carefully it has minimum side effects and sequelae. It works best when exsanguination is complete and the local anaesthetic agent is injected in a distal vein (back of the hand). Lidocaine $0.5 \%$ or prilocaine $0.5 \%(30-40 \mathrm{ml}$, or $3 \mathrm{mg}$ $\mathrm{kg}^{-1}$ ) is a suitable local anaesthetic for IVRA of the upper extremity. A larger volume (75-100 ml) with a lesser concentration $(0.25-0.35 \%)$ of local anaesthetic is necessary for the lower extremity. Bupivacaine is contraindicated, because of its potential for serious cardiotoxicity, should the local anaesthetic escape into the circulation prematurely. Chloroprocaine is contraindicated because it can cause thrombophlebitis.

For delicate hand operations, oozing from the surgical wound ('weeping') may pose some problems for the surgeon during IVRA, but this can be easily rectified if the surgeon exsanguinates the hand for a second time with a sterile Esmarch bandage just before the incision.

Although IVRA is fairly safe, it should be used carefully as it does have the potential for serious complications, including death ${ }^{55}$. The most common cause of morbidity and mortality is systemic local anaesthetic toxicity which can occur because of gross overdose of local anaesthetic agent, as a result of a tourniquet failure or abrupt removal of the tourniquet after the operation is over. Intravenous regional anaesthesia should not be used for an operation which is likely to last less than 30 min. A double tourniquet is usually applied on the upper arm, as proximal as possible; the proximal tourniquet is inflated at first. In some cases, however, it is necessary to 
switch from the proximal to the distal, when tourniquet pain becomes unbearable. This should be done very carefully, after making sure that the distal tourniquet is in fact appropriately inflated before the proximal one is released. Otherwise local anaesthetic may escape into the circulation with loss of analgesic effect and possibly causing serious systemic toxicity. Similarly, at the end of the operation, the tourniquets should be released intermittently with $10-15 \mathrm{~s}$ intervals between them.

\section{Axillary block}

Among the various types of brachial plexus block, the axillary approach is the most common for outpatient surgery. Disadvantages of axillary block include: fairly long onset time of 20-30 min, and high failure rate (15$20 \%)$. Lidocaine $(1.5 \%)$, prilocaine $(1.5 \%)$ or, in some situations, bupivacaine $(0.25-0.5 \%)$ are appropriate agents. To ensure a quicker onset and longer duration of action, some anaesthesiologists prefer to use a mixture of $2 \%$ lidocaine and $0.5 \%$ bupivacaine in equal volumes. A volume of $30-40 \mathrm{ml}$ is recommended.

Many methods of axillary block are practised. The highest success rate among these methods is the transarterial method (i.e. where the axillary artery is deliberately punctured by the advancing needle to identify the sheath around it and the injections are made just outside the arterial wall). Other methods include eliciting paraesthesia, eliciting a motor response when stimulated by a nerve stimulator attached to a sheathed needle (with a current of $0.1-0.5 \mathrm{MA}$ ), and using a perivascular approach. One strategy to convert a partially failed axillary block into a successful one is to perform additional individual nerve blocks as necessary (e.g. ulnar nerve at elbow or at wrist, musculocutaneous nerve in the belly of the coracobrachialis muscle in the axillae, intercostalbrachial nerve block in the upper arm, median nerve block at the elbow or wrist and radial nerve block at the wrist).

\section{Ankle block}

For operations on the foot, ankle block is a simple yet very effective anaesthetic technique. The major nerves supplying the foot are tibial (also called posterior tibial), deep peroneal (also called anterior tibial), superficial peroneal, saphenous and the sural nerves. These nerves can be blocked easily at the ankle, and a superficial ring block around the ankle blocks the rest of the nerves supplying the foot. Lidocaine $1 \%$ with or without epinephrine is appropriate for this block.

\section{Popliteal block}

This is a fairly easy, but rarely used, block for foot and ankle surgery. Unlike femoral-sciatic nerve blocks it does not cause prolonged weakness of the entire lower extremity. The popliteal nerves are blocked at the back of the thigh, just above the knee joint, where the sciatic nerve is divided into two parts, the tibial and the common pero- neal nerves (they are also called the medial and lateral popliteal nerves). These nerves lie next to the popliteal artery in the popliteal fossa.

\section{Ilio-inguinal and ilio-hypogastric nerve blocks}

These nerves can be blocked easily by local infiltration 3 $\mathrm{cm}$ inferior and medial to the anterior superior iliac spinous process. These are useful blocks for operations in the groin, such as inguinal hernia repair both in adults and in children. They provide good postoperative pain control for a considerable period of time.

\section{Caudal block}

Caudal blocks are commonly performed in children for operations in the groin, perineum and lower part of the body. The great advantage of a caudal block is that it provides prolonged postoperative pain relief after the operation. Bupivacaine $0.25 \%$ solution, $0.5-1.0 \mathrm{ml} \mathrm{kg} \mathrm{kg}^{-1}$ is an appropriate dose in children, depending on the level of the block intended.

\section{Knee block}

Knee block is a simple yet very successful block for knee arthroscopic procedures. After thorough aseptic precautions, the points of entry of the trocars are identified and infiltered with a local anaesthetic agent, usually lidocaine or bupivacaine. Intra-articular instillation of bupivacaine $0.5 \%(30 \mathrm{ml})$ with or without a narcotic completes the block. This block not only provides good intraoperative surgical anaesthesia, but also prolonged postoperative analgesia ${ }^{56,57}$

\section{Other peripheral blocks}

Many other less commonly used blocks including femoral-sciatic nerve block and wrist block are sometimes indicated. For ophthalmic operations, retrobulbar and peribulbar blocks are appropriate for outpatient surgery ${ }^{58}$.

\section{Spinal and epidural anaesthesia}

When indicated, the central neuroaxial blocks, such as spinal and epidural anaesthesia are appropriate for outpatient surgery. The primary objections to these blocks include patient anxiety, longer time to institute the block, possibility of dural puncture headache, backache, prolonged motor block and delay in voiding. Proper selection of the patient, explanation, psychological preparation, judicious use of pharmacological anxiolytics, innovations in timing of the block, proper selection of the local anaesthetic agent and the adjuvants, and experience and care of the anaesthesiologists should override most of these objections. 


\section{Subarachnoid vs. epidural block for outpatient surgery}

While selecting a central neuroaxial block, the possible advantages and disadvantages of subarachnoid block vs. epidural block must be considered. Compared to epidural block, subarachnoid block is simpler to perform, can be established in a shorter time, has a shorter onset time, a higher success rate and it provides a more intense analgesia and greater degree of muscle relaxation for surgery and it causes less backache. It may cause more intense sympathetic block with more profound hypotension for the same level of block. The duration of a singleshot spinal anaesthesia is restricted by the choice of the local anaesthetic agent and the additives. The continuous form of spinal anaesthesia is not appropriate for outpatient operations. As described earlier, with the introduction of the pencil-point needles (Whitacre, Sprotte), the main objection against spinal anaesthesia, postdural puncture headache, has all but disappeared even in young patients. The main advantage of epidural block over spinal is that the duration of anaesthesia may be tailored by the use of shorter-acting local anaesthetics with incremental injections via an indwelling epidural catheter. Postoperative analgesia via epidural catheter is not a practical consideration for the outpatient at this time. Thus, for outpatient surgery, at present, subarachnoid block may indeed be the preferred central neuroaxial block, unless the duration of surgery is long and unpredictable.

\section{Inappropriate regional blocks for outpatient surgery}

The regional anaesthetic techniques that are associated with possible pneumothorax are considered relative contraindications for outpatient surgery. This is because pneumothorax may not become apparent, even on chest X-ray, until several hours after the procedure. These blocks are: supraclavicular and interscalene brachial plexus blocks and intercostal blocks. Prolonged motor weakness of the lower extremity after femoral-sciatic nerve block may be a disadvantage in some situations. Subarachnoid block is no longer considered inappropriate for outpatient surgery, even in young individuals, although a continuous spinal technique should be avoided.

\section{Inappropriate patients and surgical procedures for regional anaesthesia}

Obviously, every patient is not suitable for regional anaesthetic techniques. An unwilling patient should never be 'talked into' accepting regional anaesthesia. Of course, the patient must be educated about the pros and cons of regional anaesthesia during the preoperative period, especially when a regional anaesthetic technique is clearly superior (e.g. patients with severe chronic obstructive pulmonary disease, or a patient with coronary artery disease undergoing a superficial operation in the perineum or lower extremity).

Unless there is a strong reason to avoid general anaes- thesia (e.g. severe cardiorespiratory disease), for really short outpatient surgical procedures (less than $30 \mathrm{~min}$ duration) like D\&C, cone biopsy, etc., general anaesthesia with a short-acting general anaesthetic (e.g. propofol with a laryngeal mask airway) may indeed be preferable to regional anaesthesia. Similarly, in a patient who has a back problem, spinal or epidural anaesthesia is probably not appropriate unless the risks of general anaesthesia are greater. Regional anaesthesia may often be advantageous for obese patients undergoing short peripheral operations, however, if the bony landmarks are obscure and the patient is concerned about backache, it is probably inappropriate to make innumerable attempts to perform a regional block, leaving the patient dissatisfied and permanently opposed to any regional anaesthesia in the future.

It is always important to determine the patient's coagulation status during preoperative evaluation before any regional anaesthesia is contemplated, especially a central neuroaxial block. Coagulation status is best determined by taking a history about bleeding tendency during normal day-to-day activities like easy bruising when scratched accidentally, prolonged bleeding after a small cut or frequent bleeding during brushing of teeth. When such a history is present and regional anaesthesia is still under consideration, a preoperative laboratory coagulation profile should be ordered.

\section{Discharge criteria after regional anaesthesia}

When should the outpatient receiving regional anaesthesia be discharged after surgery? The usual clinical discharge criteria (i.e. fully awake and alert, stable vital signs, absence of any respiratory distress, minimum postoperative nausea and pain) still apply for all patients whatever the type of anaesthesia received ${ }^{59}$.

For central neural blockade, it is necessary to ascertain that the autonomic and motor functions have returned to normal before discharge ${ }^{60}$. Successive measurements of arterial pressure in orthostatic conditions will detect any residual sympathetic block $^{61}$. Return of proprioception of the great toe is a good sign of adequate recovery. Most facilities require that the patient is able to void and ambulate before discharge.

Patients who receive a peripheral block (e.g. brachial plexus block) need not wait until complete recovery of the motor and sensory functions. It is imperative, however, that the limb is adequately protected by a sling and the patient and the attendant are educated about the possibility of injury to the insensitive limb from trauma and fire (e.g. from smoking and cooking).

\section{References}

1 Meridy HW. Criteria for selection of ambulatory surgery patients and guidelines for anesthetic management: A retrospective study of 1553 cases. Anesth Analg 1961; 61: $921-6$

2 Bridenbaugh LD, Soderstrom RM. Lumbar epidural block anesthesia for outpatient laparoscopy. $J$ Reprod Med 1979; 23: 85-6 
3 Bridenbaugh LD. Regional anaesthesia for outpatient surgery - a summary of 12 years experience. Can Anaesth Soc $J$ 1983; 30: 548-52

4 Randel GI, Kothary SP, Pandit SK, Brousseau M, Levy L. Recovery characteristics of three anaesthetic techniques for outpatient surgery. Amb Surg 1993; 1: 25-30

5 Dripps RD, Vandam LD. Long-term follow-up of patients who received 10,098 spinal anesthetics: Failure to discover major neurological sequelae. $J A M A$ 1954; 156: 1486-91

6 Lund PC. Peridural anaesthesia: A review of 10,000 administrations. Acta Anaesth Scand 1962; 6: 143-59

7 Usubiaga JE. Neurological complications following epidural anaesthesia. Intern Anesth Clin 1975; 13: 1-4

8 Dawkins CJM. An analysis of complications of extradural and caudal block. Anaesthesia 1969; 24: 554-63

9 Phillips OC, Ebner H, Nelson AT et al. Neurological complications following spinal anesthesia with lidocaine. A prospective study in 10,440 cases. Anesthesiology 1969; 30: $284-9$

10 de Jong RH, Haevner JE. Diazepam prevents and aborts lidocaine convulsions in monkeys. Anesthesiology 1974; 41: 226-30

11 White PF, Way WL, Trevor AJ. Ketamine - its pharmacology and therapeutic uses. Anesthesiology 1982; 56: $119-36$

12 Caplan RA, Ward RJ, Posner K, Cheney FW Unexpected cardiac arrest during spinal anesthesia: A closed claims analysis of predisposing factors. Anesthesiology 1988; 68: 5-11

13 American Society of Anesthesiologists. Standards for Basic Anesthetic Monitoring. Approved by House of Delegates on October 21, 1986 and last amended on October 13, 1993

14 Dahl JB, Schulz P, Anker-Moller E, et al. Spinal anaesthesia in young patients using a 29 -gauge needle: Technical considerations and an evaluation of postoperative complaints compared with general anaesthesia. Br J Anaesth 1990; 64: 178-82

15 Jones RJ. The role of recumbency in the prevention of post spinal headache. Anesth Analg 1974; 53: 788-96

16 Carbaat $P$, van Crevel $H$. Lumbar puncture headache: Controlled study on the preventive effect of 24 hour's bed rest. Lancet $1981 ; 2$ : 1133-5

17 Thornberry E, Thomas T: Posture and post-spinal headache. Br J Anaesth 1988; 60: 195-7

18 Flaatten H, Reader J. Spinal anaesthesia for outpatient surgery. Anaesthesia 1985; 40: 1108-11

19 Atkinson RS, Lee JA. Spinal anaesthesia and day case surgery? Anaesthesia 1985; 40: 1059-60

20 Clarke GA, Power KJ. Spinal anaesthesia for day case surgery. Ann Roy Coll Surg 1988; 70: 144-6

21 Perz RR, Johnson DI, Shinozaki T. Spinal anaesthesia for outpatient surgery. Anesth Analg 1988; 67: S168

22 Milroy MF, Neal JM, Bridenbaugh LD. Is post spinal headache more frequent in outpatients? Reg Anesth 1989; 14: 2

23 Neal JM, Bridenbaugh LD, Mulroy MF. Incidence of postdural puncture headache is similar between $22 \mathrm{G}$ Greene and 26G Quincke spinal needles. Anesthesiology 1989; 71: A678

24 Vandam LD, Dripps RD. Long-term follow-up of patients who received 10,098 spinal anesthetics. III Syndrome of decreased intracranial pressure (headache and ocular difficulties) $J A M A$ 1956; 161: 586-91

25 Gielen M. Postdural puncture headache (PDPH): A review. Reg Anesth 1989; 14: 101-6

26 Mihic DN. Post spinal headache and relationship of needle bevel to longitudinal dural fibers. Reg Anesth 1985; 10: $76-81$

27 Hatfalvi BI. The dynamics of post spinal headache. Headache 1977; 17:64 6
28 Hart JR, Whitacre RJ. Pencil point needle in prevention of spinal headache. $J A M A 1981 ; 147: 657-8$

29 Sprotte G, Schedel R, Pajunk H. Eine 'atraumatische' Universalkanule fur einzeitige Regionalanaesthesien. Reg Anesth 1987; 10: 104-8

30 Lim M, Cross GD, Sold M. Post spinal headache. A comparison of a $24 \mathrm{G}$ Sprotte needle and a $29 \mathrm{G}$ Quincke needle. Anaesthetist 1992; 41: 539-43

31 Kang SB, Goodnaugh DE, Lee YK et al. Comparison of 26- and 27-G needles for spinal anesthesia for ambulatory surgery patients. Anesthesiology 1992; 76: 734-8

32 Lynch J, Krings-Ernst K, Topalidis K et al. Use of 25 gauge Whitacre needle to reduce the incidence of postdural puncture headache. $B r J$ Anaesth 1991; 67 : $690-3$

33 Seeberger MD, Mathis LL, Dreve $\mathbf{J}$ et al. Comparison of spinal, and epidural anesthesia for patients younger than 50 years of age. Anesth Analg 1994; 78: 667-73

34 Brown EM, Elman DS. Postoperative backache. Anesth Analg 1961; 40: 683

35 Barsa J, Batra M, Fink BR et al. A comparative in vitro study of local neurotoxicity of lidocaine, bupivacaine, 2-chloroprocaine, and a mixture of 2-chloroprocaine and bupivacaine. Anesth Analg 1982; 61: 961-7

36 Fibuch EE, Opper SE. Back pain following epidurally administered Nesacaine-MPF. Anesth Analg 1989; 69: 113-15

37 Levy L, Randel GI, Pandit SK. Does chloroprocaine (Nesacaine MPF) for epidural anesthesia increase the incidence of backache? Letter. Anesth Analg 1989; 71: 476

38 Stevens RA, Chester WL, Artuso JD et al. Back pain after epidural anesthesia with chloroprocaine in volunteers: Preliminary report. Reg Anesth 1991; 16: 199-203

39 Foster AH, Carleson BM. Myotoxicity of local anesthetics and regeneration of the damaged muscle fibers. Anesth Analg 1980; 59: 727-36

40 Covino BG, Marx GF, Finster $M$ et al. Prolonged sensory/motor deficits following inadvertent spinal anaesthesia. Anesth Analg 1980; 59: 399-400

41 Geissen AJ, Datta S. Lambert D. The chloroprocaine controversy II: Is chloroprocaine neurotoxic? Reg Anesth 1984; 9: $135-45$

42 de Jong RH. Last round for a 'Heavyweight'? Anesth Analg 1994; 78: 3-4

43 Schneider M, Ettlin T, Kaufmann M et al. Transient neurological toxicity after hyperbaric subarachnoid anesthesia with 5\% lidocaine. Anesth Analg 1993; 76: 1154-7

44 Hurley RJ, Lambert DH. Continuous spinal anesthesia with micro catheters technique. Preliminary report. Anesth Analg 1990; 70: 97-102

45 Reigler ML, Drasner K, Krejcie TC et al. Cauda equina syndrome after continuous spinal anesthesia. Anesth Analg 1991; 72: 275-81

46 Lambert DH, Hurley RJ. Cauda equina syndrome and continuous spinal anesthesia. Anesth Analg 1991; 72: $817-20$

47 Ross BK, Coda B, Heath CH. Local anesthetic distribution in a spinal model: a possible mechanism of neurological injury after continuous spinal anesthesia. Reg Anesth 1992; 17: 69-77

48 Van Gessel EF, Proplan J, Fuchs T et al. Influence of injection of speed on the subarachnoid distribution of isobaric bupivacaine 0.5\%. Anesth Analg 1993; 77: 483-7

49 Arthur GR, Covino BG. What's new in local anesthetics? Anesth Clin North Amer 1988; 6: 357-70

50 DiFazio CA, Rowlingson JC, Moscicki JC. pH adjustment of local anesthetics. Reg Anesth 1994; 19: 70

51 St. Amand, M, Allen GC, Lui A et al. Intraarticular 
morphine and bupivacaine for analgesia following outpatient arthroscopic knee surgery. Anesthesiology 1992; 77: A817

52 Bjornsson A, Gupta A, Vegfors $M$ et al. Intraarticular morphine for postoperative analgesia following knee arthroscopy. Reg Anesth 1994; 19: 104-8

53 Cousins MJ, Bridenbaugh PO. Neural Blockade, Philadelphia: JB Lippincott Co., 1980

$54 \mathrm{Katz}$ J. Atlas of regional Anesthesia 2 nd edn. Norwalk, CT: Appelton \& Lange, 1993

55 Heath ML. Deaths following intravenous regional anaesthesia. $\mathrm{Br}$ Med $J$ 1984; 285: 913-14

56 Carnes RS, Butterworth JF, Poeling GS et al. Safety and efficacy of intraarticular bupivacaine and epinephrine anesthesia for knee arthroscopy. Anesthesiology 1989; 71 A729
57 Dahl MR, Dasta JF, Zuelzer WA et al. Arthroscopic knee surgery under lidocaine local anesthesia. Anesthesiology 1989; 71: A730

58 Ali-Melkkila TM, Virkkila M, Jyrkkio H. Regional anesthesia for cataract surgery: Comparison of retrobulbar and peribulbar techniques. Reg Anesth 1992; 17: 219-22

59 Wetchler BV. Postoperative management, discharge, and follow-up. Anesth Clin N Am 1987; 5: 113-36

60 Pflug AE, Aasheim GM, Foster C. Sequence of return of neurological function and criteria for safe ambulation following subarachnoid block (spinal anaesthesia). Can Anaesth Soc J 1978; 25: 133-9

61 Alexander $\mathrm{C}$, Teller L, Gross J et al. New discharge criteria decrease recovery room time after subarachnoid block. Anesthesiology 1989; 70: 640-3
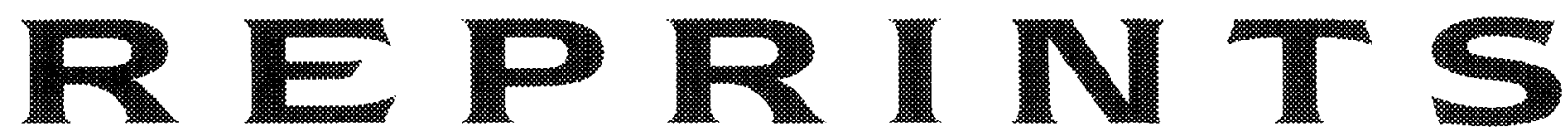

We offer a reprints service in respect of all articles in this journal.

Companies: an article featuring your product or a new application provides its readers with an independent authoritative comment.

Reprints can be used by you to inform and educate your customers and staff about scientific or technical advances in subjects relevant to your

business. They are a cost-effective, ethical way of promoting your company and its products.

For a quotation on your reprint requirements, please contact:

The Reprints Department, Butterworth-Heinemann Ltd., Linacre House, Jordan Hill, Oxford, OX2 8DP. Tel: +44 (O)865 310366. Fax: + 44 (O)865 314519

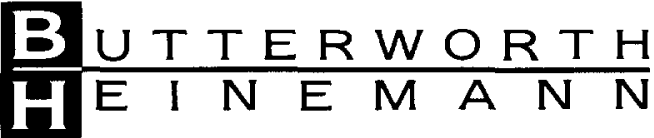

